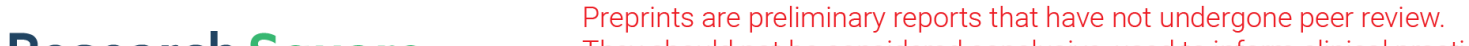 They should not be considered conclusive, used to inform clinical practice, or referenced by the media as validated information. \\ Fixed-Charge Solid Transportation Problem with Budget Constraints Based on Carbon Emission in Neutrosophic Environment
}

\section{Shyamali Ghosh}

Dept. of Applied Matjematics, Vidyasagar University

\section{Sankar Kumar Roy ( $\nabla$ sankroy2006@gmail.com )}

Dept. of Applied Mathematics, Vidyasagar University Jose Luis Verdegay

Dept. of Computer Science and Artificial Intelligence, University of Granada.

\section{Research Article}

Keywords: Fixed-charge transportation problem, Multi-objective decision making, Carbon emission, Neutrosophic linear programming, Fuzzy programming, Global criterion method, Compromise solution

Posted Date: August 3rd, 2021

DOI: https://doi.org/10.21203/rs.3.rs-705598/v1

License: (9) This work is licensed under a Creative Commons Attribution 4.0 International License. Read Full License 


\title{
Fixed-charge solid transportation problem with budget constraints based on carbon emission in neutrosophic environment
}

\author{
Shyamali Ghosh $^{1, \dagger} \cdot$ Sankar Kumar Roy ${ }^{1, *} \cdot$ José Luis Verdegay ${ }^{2}$ \\ Received: date / Accepted: date
}

\begin{abstract}
This paper is to integrate among solid transportation problem, budget constraints and carbon emission with probable maximum profit. The limits of air pollution and climate variation are solely dependent by exerting $\mathrm{CO}_{2}$ gas and rest greenhouse gases due to myriad transportation system. Henceforth, it is our apt mission to minimize carbon emission for pollution free environment. Again transportation system with single objective is hardly applicable to the situation with more than one criterion. Therefore multi- objective decision making is incorporated for designing reallife transportation problem. Due to time pressure, data limitation, lack of information or measurement errors in practical problems, there exist some hesitations or suspicions. Based on the fact, decision maker considers indeterminacy in the designed problems. To overcome the restriction on occurrence and non-occurrence of fuzzy and intuitionistic fuzzy, neutrosophic set is very important and suitable to accommodate such general structure of problems. Therefore neutrosophic environment with neutrosophic linear programming, fuzzy programming and global criterion method are profiled to search the compromise solution of the multi- objective transportation problem (MOTP). Thereafter, the performance of the considered model is useful by evaluating a numerical example; and then the derived results are compared. Finally sensitivity analysis and conclusions with upcoming works of this research are stated hereafter.
\end{abstract}

\footnotetext{
${ }^{1}$ Department of Applied Mathematics with Oceanology and Computer Programming, Vidyasagar University,

Midnapore-721102, West Bengal, India.

$\dagger$ E-mail: shyamalighosh1989@gmail.com

*E-mail: sankroy2006@gmail.com

${ }^{*}$ Corresponding Author

* Orcid: 0000-0003-4478-1534

${ }^{2}$ Department of Computer Science and Artificial Intelligence,

University of Granada, Granada, Spain

${ }^{2}$ E-mail: verdegay@decsai.ugr.es
}

Keywords Fixed-charge transportation problem - Multiobjective decision making $\cdot$ Carbon emission $\cdot$ Neutrosophic linear programming $\cdot$ Fuzzy programming $\cdot$ Global criterion method $\cdot$ Compromise solution

\section{Introduction}

Only a single objective function is considered to the customary transportation system in the last few decades. There subsist more than the single criterion such as transportation cost, average delivery time of product, deterioration rate of goods, fixed-charge for an open route etc. at the time of passing a homogeneous product from a source to different destinations in the avenue of competitive economic condition. For this sake, the single objective transportation problem (TP) is not enough to tractable such real-life decision making problem. To get rid of such situation, the multi-objective decision making is included in the traditional TP where the objective functions are contrary to each other. Hitchcock (1941) first defined TP and then TP with linear programming is known as Hitchcock-Koopmans TP. There exist various research papers on TP with multi-objective nature in crisp or imprecise data. A few of them is presented including their works. Maity et al. (2019) analyzed multimodal TP and its application to artificial intelligence. Ebrahimnejad (2014) represented a new approach for solving TP with generalized trapezoidal fuzzy numbers. MOTP with fuzzy environment was provided by Li and Lai (2000). Maity et al. (2016) designed MOTP in uncertain environment with cost reliability. An extension of general TP is fixed-charge transportation problem (FTP) with the transported fixed-charge being independent of its amount. A fixed cost is called set up cost bearing the cost of transportation is considered while the solution appears with the level of positivity and hence such a problem is named as FTP and it is associated with $0-1$ variable. There 
comes a cumulation of some charges such as renting cost of vehicle, landing fees at an airport, toll charges on a high way, establishment cost for machines. All these costs are considered for the transportation of some quantity towards destinations. Adding to all the cost, the TP is called FTP and two sets of cost are there with FTP. The names of these costs are direct cost and fixed-charge. The association of direct cost for each source to destination and fixed-charge takes place when the function of transportations is there in the corresponding source-destination pair. Hirsch and Dantzig (1968) first defined FTP. Besides, there are some exact methods provided by literature review on FTP. Midya and Roy (2017) represented an application of interval programming using interval and rough interval on FTP in different environments. Midya and Roy (2014) included fixed-charge MOTP with single-sink and multi-index stochastic environment. Roy et al. (2018) used random rough variables for solving MOTP with fixed-charge.

The TP is renamed as solid transportation problem (STP) which was first introduced by Haley (1962), when adds conveyance constraints with source constraints and destination constraints. Goods train, ships, trucks, cargo flights are the media for the transportation of homogeneous products from sources to destinations. The TP is restructured and renamed as FSTP after the single type conveyance is used in FTP. Roy and Midya (2019) solved FSTP in intuitionistic fuzzy (IF) environment with multi-objective and product blending concept. Das et al. (2020) represented STP and facility location with carbon policies. Ghosh et al. (2021) solved a multi-objective STP in which variables and parameters are IF in nature. Roy et al. (2019) analyzed FSTP with multiitem and multi- objective using two-fold uncertainty.

In previous research works, STP involves with sources, demands and conveyance capabilities for solving decision making problem. But no one can work with STP under budget constraints including fixed-charge, purchasing cost, transportation cost and profit maximization. Also budget is an important fact in transportation problem. Whenever transporting distinct items, then transportation cost rapidly increases, which effects on the whole system. Again considering the budget, we optimize the several objective functions which are conflicting to each other. These objective functions minimize total transportation cost, total delivery time, deterioration charge of breakable goods, as a result the total profit is to be maximized. Many researchers applied budget constraints in MOTP. Majumder et al. (2019) used budget constraints in a FSTP with uncertain multi-objective and multiitem environment. Das et al. (2016) analyzed Gaussian type2 fuzzy for solving STP with breakable multi-item multistage under budget. Ghosh and Roy (2021) formulated and solved multi-objective product blending fixed-charge transportation problem with truck load constraints through transfer under fuzzy-rough environment. Sifaouil and Aider
(2020) considered budget constraints and safety measure in multi-objective multi-item fixed charge solid transportation problem under uncertain interval programming environment. Most of the cases, the transportation sector transports items and passengers by bus, train, truck, car, ship, flight etc. Since internal combustion of engines causes the emission of green house gasses, therefore the transportation system is mostly involved with emission of $\mathrm{CO}_{2}$ gas and other green house gasses. The half part of green house gasses emits from lightduty vehicles, passenger car, minibus etc. and the remaining part emits from heavy-duty vehicles such as truck, ships, freight transport etc. The greenhouse gas emission is highrisk for the environmental and air pollution. The carbon emission depends on its fuel type, engine type, traffic rule, road condition, driving rules, etc. Many researchers studied on carbon emission in various environments. Here we include some research works on carbon emission in transportation poblem. Ding et al. (2013) analyzed about carbon emission reduction and its potential in China for transportation. Sengupta et al. (2018) solved a STP with carbon emission using Gamma type-2 defuzzification approach. Song and Leng (2012) analyzed carbon emission policies for single-period problem. Tarulescu et al. (2017) worked for $\mathrm{CO}_{2}$ emission reduction strategies on smart transportation. Midya et al. (2021) presented a multi-stage multi-objective FSTP in a green supply chain.

Neutrosophic set is the generalized extension of fuzzy set (Zadeh (1965)) and intuitionistic fuzzy set (IFS) (Atanassov (1986)), which was first studied by Smarandache (1999). To overcome the restriction on occurrence and non-occurrence of fuzzy and intuitionistic fuzzy, neutrosophic set is very important and suitable that provides the general structure. Meaning of neutrosophic set is neutral and it is based on the logic of universe that the elements presented by three degrees. These are truth degree, indeterminacy degree and falsity degree and they lie in $[0,1]$. Neutrosophic set differs from IFS which involves only the uncertainty of truth and falsity, that is belongingness and non-belongingness degrees. But in neutrosophic, indeterminacy factor occurs, which is independent on truth value and falsity value, and indeterminacy is quantified explicitly that provides more additional information of fuzzy concept. Smarandache first introduced the concept of indeterminacy and denoted by I. The neutrosophic number $(\mathrm{NN})$ is presented by $\mathrm{z}=\mathrm{p}+\mathrm{Iq}$, for $\mathrm{p}, \mathrm{q}$ $\in \mathbb{R}$ and $\mathrm{I}$ is the indeterminacy, which gives indeterminate value. Here $\mathrm{p}$ is the determinate part and $\mathrm{qI}$ is the indeterminate part. Therefore neutrosophic is a useful mathematical concept for describing incomplete and indeterminate information. In the case of time pressure, measurement errors, limited data or lack of information, the practical problems are solved with help of indeterminacy I. Therefore it is more important to define NN that contains indeterminacy I and handles all the programming problems with indetermi- 
nate environment. To control over any indeterminate problems, there are also introduced neutrosophic function, neutrosophic pre-calculus, neutrosophic calculus by Smarandache in 2015. Some recent studies on neutrosophic set are included here. Rizk-Allah et al. (2018) represented a MOTP and solved in neutrosophic environment. Time-neutrosophic soft set studied by Khalil et al. (2018) and analyzed its application. Das and Roy (2019) analyzed the impact of $\mathrm{CO}_{2}$ in an integrated transportation and facility location problem under neutrosophic environment. Ye et al. (2018) analyzed non-linear programming problems and solution methods in neutrosophic number environment. The main contributions of this paper are as follows:

(i) In this MOTP, all the parameters are considered as NNs for controlling the indeterminacy.

(ii) For neutrosophic system, there exist truth membership, indeterminacy membership and falsity membership functions which help the problem for searching good solution.

(iii) Objective functions are conflicting to each other. Therefore for finding the compromise solution we use three methods, namely neutrosophic linear programming, fuzzy programming and global criterion method.

(iv) Neutrosophic linear programming always increases the degree of truth membership value, indeterminacy membership value and at the same time decreases the degree of falsity membership value.

(v) Carbon emission system is more important factor in transportation system. Minimization of carbon emission charge directly maximizes the profit of the system as it helps to minimize the total transportation cost and decreases the rate of air pollution indirectly.

(vi) Budget constraints and carbon capacity maximize the profit and minimize the deterioration.

(vii) Sensitivity analysis is introduced for analyzing the effect of change of coefficient and finding the ranges of all parametric values.

The remaining part of the paper is structured as follows: Some basic definitions on neutrosophic set are presented in Section 2. In Section 3, the mathematical model and three procedures are discussed. Section 4 introduces the solution methodology. Sections 5 and 6 describe the drawbacks of existing methods and advantages of our proposed method respectively. Numerical example with results and discussion are given in Section 7. Section 8 depicts the sensitivity analysis of the parameters. Conclusions and future research scopes are provided in Section 9.

\section{Preliminaries}

We recall here some basic definitions of neutrosophic set which are mostly useful in formulating the proposed problem.
The concept of neutrosophic set is the analytical sight to represent the indeterminate and inconsistent information and applied in scientific and engineering applications.

Definition 2.1: Neutrosophic set: Wang et al. (2010) Let $X$ be the universal set. A single valued neutrosophic set $\tilde{A}^{n}$ over $X$ is of the form $\tilde{A}^{n}=\left\{<x, \mu_{\tilde{A}^{n}}(x), \sigma_{\tilde{A}^{n}}(x), \gamma_{\tilde{A}^{n}}(x)>: x \in\right.$ $X\}$, where $\mu_{\tilde{A}^{n}}(x): X \rightarrow[0,1], \sigma_{\tilde{A}^{n}}(x): X \rightarrow[0,1], \gamma_{\tilde{A}^{n}}(x):$ $X \rightarrow[0,1]$ with $0 \leq \mu_{\tilde{A}^{n}}(x)+\sigma_{\tilde{A}^{n}}(x)+\gamma_{\tilde{A}^{n}}(x) \leq 3, \forall x \in X$. Here $\mu_{\tilde{A}^{n}}(x), \sigma_{\tilde{A}^{n}}(x)$ and $\gamma_{\tilde{A}^{n}}(x)$ are the degrees of truthmembership, indetermin-acy-membership and falsity membership of $x$ in $\tilde{A}^{n}$ respectively.

Definition 2.2: The general form of a multi-objective optimization problem with $l$ objective functions, $m$ constraints and $n$ variables is given as follows:

$$
\begin{array}{ll}
\operatorname{minimize} & Z(X)=\left(Z_{1}(x), Z_{2}(x), \ldots, Z_{l}(x)\right) \\
\text { subject to } & g_{j}(X) \leq 0, \quad(j=1,2, \ldots, m) \\
& x_{i} \geq 0, \quad(i=1,2, \ldots, n), x_{i} \in X \subseteq \mathbb{R}^{n} .
\end{array}
$$

The truth-membership $\mu_{l}^{I}\left(Z_{l}\right)$, indeterminacy-membership $\sigma_{l}^{I}\left(Z_{l}\right)$ and falsity-membership $v_{l}^{I}\left(Z_{l}\right)$ functions for the objective function $Z_{l}$ are respectively defined as:

$$
\begin{gathered}
\mu_{l}^{I}\left(Z_{l}\right)= \begin{cases}1, & \text { if } Z_{l} \leq t_{l}, \\
1-\frac{Z_{l}-t_{l}}{a_{l}}, & \text { if } t_{l} \leq Z_{l} \leq t_{l}+a_{l}, \\
0, & \text { if } Z_{l} \geq t_{l}+a_{l},\end{cases} \\
\sigma_{l}^{I}\left(Z_{l}\right)= \begin{cases}0, & \text { if } Z_{l} \leq t_{l}, \\
\frac{Z_{l}-t_{l},}{d_{l},} & \text { if } t_{l} \leq Z_{l} \leq t_{l}+d_{l}, \\
1-\frac{Z_{l}-t_{l}}{a_{l}-d_{l},}, & \text { if } t_{l}+d_{l} \leq Z_{l} \leq t_{l}+a_{l}, \\
0, & \text { if } Z_{l} \geq t_{l}+a_{l},\end{cases}
\end{gathered}
$$$$
v_{l}^{I}\left(Z_{l}\right)= \begin{cases}0, & \text { if } \quad Z_{l} \leq t_{l}, \\ \frac{Z_{l}-t_{l}}{c_{l}}, & \text { if } t_{l} \leq Z_{l} \leq t_{l}+c_{l}, \\ 1, & \text { if } \quad Z_{l} \geq t_{l}+c_{l} .\end{cases}
$$

Where $t_{l}$ is the target value for $Z_{l}$ and $a_{l}, d_{l}$ and $c_{l}$ are the acceptance tolerance, indeterminacy tolerance and rejection tolerance respectively. The graphical presentation of three membership functions is shown in Fig. 1.

\section{Definition 2.3: Interval valued neutrosophic number:}

Smarandache (2013) An neutrosophic number is denoted by $\tilde{a}^{n}=p+q I$, where $p, q \in \mathbb{R}$ and $I \subseteq[0,1]$ is the indeterminacy. Now $I=\left[I^{L}, I^{U}\right]$, and therefore the interval form of $\tilde{a}^{n}=\left[p+q I^{L}, p+q I^{U}\right]=\left[a^{L}, a^{U}\right]$.

Basic properties of interval valued neutrosophic number: Let $\tilde{a}_{1}^{n}=p_{1}+q_{1} I_{1}$ and $\tilde{a}_{2}^{n}=p_{2}+q_{2} I_{2}$ with $I_{1}=\left[I_{1}^{L}, I_{1}^{U}\right]$, $I_{2}=\left[I_{2}^{L}, I_{2}^{U}\right]$ be two neutrosophic numbers. The interval forms are $\tilde{a}_{1}^{n}=\left[p_{1}+q_{1} I_{1}^{L}, p_{1}+q_{1} I_{1}^{U}\right]=\left[a_{1}^{L}, a_{1}^{U}\right]$ and $\tilde{a}_{2}^{n}=\left[p_{2}+\right.$ $\left.q_{2} I_{2}^{L}, p_{2}+q_{2} I_{2}^{U}\right]=\left[a_{2}^{L}, a_{2}^{U}\right]$. Now the basic properties are defined as:

1. Addition: $\tilde{a}_{1}^{n}+\tilde{a}_{2}^{n}=\left[a_{1}^{L}+a_{2}^{L}, a_{1}^{U}+a_{2}^{U}\right]$.

2. Subtraction: $\tilde{a}_{1}^{n}-\tilde{a}_{2}^{n}=\left[a_{1}^{L}-a_{2}^{U}, a_{1}^{U}-a_{2}^{L}\right]$. 


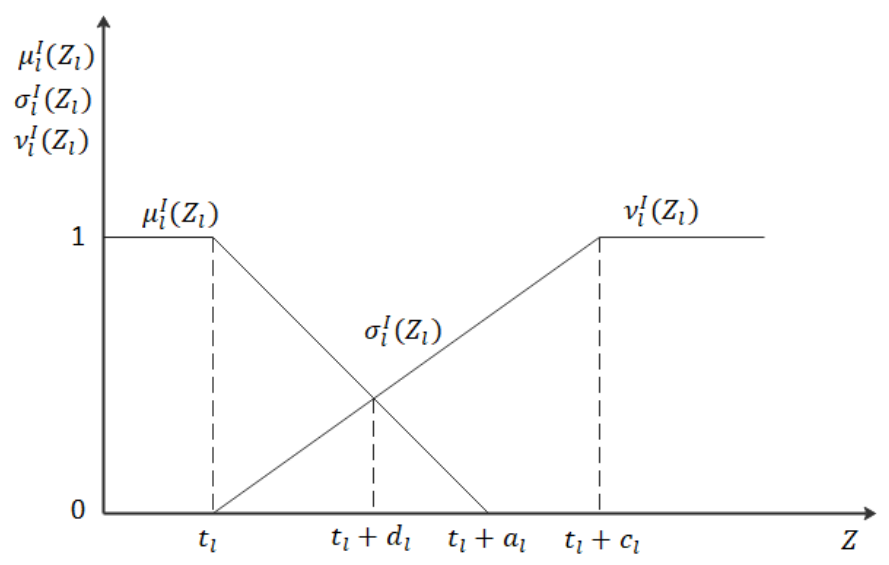

Fig 1: Truth-membership, indeterminacy-membership and falsity-membership for $Z_{l}$.

3. Multiplication: $\tilde{a}_{1}^{n} * \tilde{a}_{2}^{n}=\left\{\begin{array}{l}{\left[\min \left(a_{1}^{L} * a_{2}^{L}, a_{1}^{L} * a_{2}^{U}, a_{1}^{U} * a_{2}^{L},\right.\right.} \\ \left.a_{1}^{U} * a_{2}^{U}\right), \\ \max \left(a_{1}^{L} * a_{2}^{L}, a_{1}^{L} * a_{2}^{U}, a_{1}^{U} * a_{2}^{L},\right. \\ \left.\left.a_{1}^{U} * a_{2}^{U}\right)\right] .\end{array}\right.$

4. Division: $\tilde{a}_{1}^{n} / \tilde{a}_{2}^{n}=\left\{\begin{array}{l}{\left[\min \left(a_{1}^{L} / a_{2}^{L}, a_{1}^{L} / a_{2}^{U}, a_{1}^{U} / a_{2}^{L}, a_{1}^{U} / a_{2}^{U}\right),\right.} \\ \left.\max \left(a_{1}^{L} / a_{2}^{L}, a_{1}^{L} / a_{2}^{U}, a_{1}^{U} / a_{2}^{L}, a_{1}^{U} / a_{2}^{U}\right)\right], \\ 0 \notin \tilde{a}_{2}^{n} .\end{array}\right.$

5. Scalar multiplication: $k \cdot \tilde{a}^{n}=\left\{\begin{array}{l}{\left[k a^{L}, k a^{U}\right], \text { if } k \geq 0,} \\ {\left[k a^{U}, k a^{L}\right] \text {, if } k<0 .}\end{array}\right.$

6. Absolute value: $\left|\tilde{a}^{n}\right|= \begin{cases}{\left[a^{L}, a^{U}\right],} & \text { if } \alpha^{L} \geq 0, \\ {\left[0, \max \left(-a^{L}, a^{U}\right)\right],} & \text { if } a^{L} \leq 0 \leq \\ & a^{U}, \\ {\left[-a^{U},-a^{L}\right],} & \text { if } a^{U} \leq 0 .\end{cases}$

7. Inequality: Ishibuchi and Tanaka (1990)

$$
\begin{cases}x \leq\left[a^{L}, a^{U}\right] \equiv \exists z \in\left[a^{L}, a^{U}\right] \text { and } x \leq z, \\ x \geq\left[a^{L}, a^{U}\right] \equiv \exists z \in\left[a^{L}, a^{U}\right] \text { and } x \geq z \\ {\left[a^{L} \leq b^{L}, a^{U} \leq b^{U}\right],} & \text { iff }\left[a^{L}, a^{U}\right] \leq\left[b^{L}, b^{U}\right], \\ {\left[a^{L} \geq b^{L}, a^{U} \geq b^{U}\right],} & \text { iff }\left[a^{L}, a^{U}\right] \geq\left[b^{L}, b^{U}\right] .\end{cases}
$$

Definition 2.4: Neutrosophic linear programming problem: Ye (2018) A neutrosophic linear programming problem is a general optimization problem if the following conditions are met:

1. The neutrosophic objective function is linear.

2. The decision variables are all non-negative.

3. The structural constraints are all of the types of " $\leq$ " or " $\geq$ ".

Definition 2.5: Constrained Neutrosophic optimization problem: Ye et al. (2018) In general, a constrained optimization problem in $n$ decision variables with NNs is de- fined as follows:

minimize/maximize $F(x, I)$

$$
\begin{array}{ll}
\text { subject to } & g_{i}(x, I) \leq 0, i=1,2, \ldots, m \\
& h_{j}(x, I) \geq 0, j=1,2, \ldots, l \\
& p_{k}(x, I)=0, k=1,2, \ldots, o \\
& x \in Z^{n}
\end{array}
$$

When the indeterminacy $I$ is considered as a possible interval range, the optimal solution of all feasible intervals forms the feasible region or feasible set for $x$ and $I=\left[I^{L}, I^{U}\right]$. In this case, the value of the NN objective function is an optimal possible interval (NN) for $F(x, I)$. For example, let us consider the following optimization problem with $I=[0,1]$ :

$$
\begin{array}{ll}
\operatorname{maximize} F(x, I) & =(2+3 I) x_{1}+(4+I) x_{2} \\
& =\left[2 x_{1}+4 x_{2}, 5 x_{1}+5 x_{2}\right] \\
\text { subject to } & (1+I) x_{1}+(2+I) x_{2} \leq(6+2 I) \\
& =\left[x_{1}+2 x_{2}, 2 x_{1}+3 x_{2}\right] \leq[6,8] \\
& (2+3 I) x_{1}+(3+I) x_{2} \leq(8+2 I) \\
& =\left[2 x_{1}+3 x_{2}, 5 x_{1}+4 x_{2}\right] \leq[8,10]
\end{array}
$$

Now, this problem can be transformed into two equivalent crisp sub-problems to obtain worst (lower bound) and best (upper bound) solutions as follows:

\section{Sub-problem 1:}

maximize $2 x_{1}+4 x_{2}$

subject to $x_{1}+2 x_{2} \leq 6$

\section{Sub-problem 2:}

$$
2 x_{1}+3 x_{2} \leq 8
$$

$$
\begin{array}{ll}
\operatorname{maximize} & 5 x_{1}+5 x_{2} \\
\text { subject to } & x_{1}+2 x_{2} \leq 6 \\
& 2 x_{1}+3 x_{2} \leq 8
\end{array}
$$

By solving two sub-problems we get the optimal solution (as NN) of the original problem as: $F=[10.67,20]$.

Definition 2.6: Smarandache (1999) Let two single valued neutrosophic sets $\tilde{A}^{n}$ and $\tilde{B}^{n}$ over the universal set $X$ are of the form $\tilde{A}^{n}=\left\{<x, \mu_{\tilde{A}^{n}}(x), \sigma_{\tilde{A}^{n}}(x), \gamma_{\tilde{A}^{n}}(x)>: x \in X\right\}$, $\tilde{B}^{n}=\left\{<x, \mu_{\tilde{B}^{n}}(x), \sigma_{\tilde{B}^{n}}(x), \gamma_{\tilde{B}^{n}}(x)>: x \in X\right\}$. Then some basic properties are defined as:

1. $\tilde{A}^{n} \subset \tilde{B}^{n}$ if and only if $\mu_{\tilde{A}^{n}}(x) \leq \mu_{\tilde{B}^{n}}(x), \sigma_{\tilde{A}^{n}}(x) \leq \sigma_{\tilde{B}^{n}}(x)$, $\gamma_{\tilde{A}^{n}}(x) \geq \gamma_{\tilde{B}^{n}}(x)$.

2. $\tilde{A}^{n}=\tilde{B}^{n}$ if and only if $\mu_{\tilde{A}^{n}}(x)=\mu_{\tilde{B}^{n}}(x), \sigma_{\tilde{A}^{n}}(x)=\sigma_{\tilde{B}^{n}}(x)$, $\gamma_{\tilde{A}^{n}}(x)=\gamma_{\tilde{B}^{n}}(x)$.

3. The complement of $\tilde{A}^{n}$ is denoted by $\left(\tilde{A}^{n}\right)^{c}$ and defined by $\left(\tilde{A}^{n}\right)^{c}=\left\{<x, \gamma_{\tilde{A}^{n}}(x)\right.$, $\left.1-\sigma_{\tilde{A}^{n}}(x), \mu_{\tilde{A}^{n}}(x)>: x \in X\right\}$.

4. The intersection of $\tilde{A}^{n}$ and $\tilde{B}^{n}$ is defined by $\tilde{A}^{n} \cap \tilde{B}^{n}=$ $\left\{<x, \min \left\{\mu_{\tilde{A}^{n}}(x), \mu_{\tilde{B}^{n}}(x)\right\}\right.$, $\left.\min \left\{\sigma_{\tilde{A}^{n}}(x), \sigma_{\tilde{B}^{n}}(x)\right\}, \max \left\{\gamma_{\tilde{A}^{n}}(x), \gamma_{\tilde{B}^{n}}(x)\right\}>: x \in X\right\}$. 
5. The union of $\tilde{A}^{n}$ and $\tilde{B}^{n}$ is defined by $\tilde{A}^{n} \cup \tilde{B}^{n}=\{<$ $x, \max \left\{\mu_{\tilde{A}^{n}}(x), \mu_{\tilde{B}^{n}}(x)\right\}, \max$

$\left.\left\{\sigma_{\tilde{A}^{n}}(x), \sigma_{\tilde{B}^{n}}(x)\right\}, \min \left\{\gamma_{\tilde{A}^{n}}(x), \gamma_{\tilde{B}^{n}}(x)\right\}>: x \in X\right\}$.

Definition 2.7: Smarandache (1999) Let $\tilde{A}^{n}=\left\{<x, \mu_{\tilde{A}^{n}}(x)\right.$, $\left.\sigma_{\tilde{A}^{n}}(x), \gamma_{\tilde{A}^{n}}(x)>: x \in X\right\}$ and $\tilde{B}^{n}=\left\{<x, \mu_{\tilde{B}^{n}}(x), \sigma_{\tilde{B}^{n}}(x), \gamma_{\tilde{B}^{n}}(x)\right.$ $>: x \in X\}$ are two single valued neutrosophic sets. Some operations of neutrosophic sets are defined as:

1. $\lambda \tilde{A}^{n}=<1-\left(1-\mu_{\tilde{A}^{n}}(x)\right)^{\lambda}, \sigma_{\tilde{A}^{n}}(x)^{\lambda}, \gamma_{\tilde{A}^{n}}(x)^{\lambda}>; \lambda \geq 0$.

2. $\left(\tilde{A}^{n}\right)^{\lambda}=<\mu_{\tilde{A}^{n}}(x)^{\lambda}, 1-\left(1-\sigma_{\tilde{A}^{n}}(x)\right)^{\lambda}, 1-\left(1-\gamma_{\tilde{A}^{n}}(x)\right)^{\lambda}$ $>; \lambda \geq 0$.

3. $\tilde{A}^{n}+\tilde{B}^{n}=<\mu_{\tilde{A}^{n}}(x)+\mu_{\tilde{B}^{n}}(x)-\mu_{\tilde{A}^{n}}(x) \mu_{\tilde{B}^{n}}(x), \sigma_{\tilde{A}^{n}}(x)$ $\sigma_{\tilde{B}^{n}}(x), \gamma_{\tilde{A}^{n}}(x) \gamma_{\tilde{A}^{n}}(x)>$.

4. $\tilde{A}^{n} \cdot \tilde{B}^{n}=<\mu_{\tilde{A}^{n}}(x) \mu_{\tilde{B}^{n}}(x), \sigma_{\tilde{A}^{n}}(x)+\sigma_{\tilde{B}^{n}}(x)-\sigma_{\tilde{A}^{n}}(x) \sigma_{\tilde{B}^{n}}(x)$, $\gamma_{\tilde{A}^{n}}(x)+\gamma_{\tilde{B}^{n}}(x)-\gamma_{\tilde{A}^{n}}(x) \gamma_{\tilde{B}^{n}}(x)>$.

Abbreviations: Here we display all the abbreviations which are used in this paper.

$\begin{array}{ll}\text { MOTP } & \text { multi-objective transportation problem } \\ \text { TP } & \text { transportation problem } \\ \text { FTP } & \text { fixed-charge transportation problem } \\ \text { STP } & \text { solid transportation problem } \\ \text { FSTP } & \text { fixed-charge solid transportation problem } \\ \text { IF } & \text { intuitionistic fuzzy } \\ \text { IFS } & \text { intuitionistic fuzzy set } \\ \text { NN } & \text { neutrosophic number } \\ \text { MFSTP } & \text { multi-objective fixed-charge solid transportation } \\ & \text { problem } \\ \text { NLPP } & \text { neutrosophic linear programming problem } \\ \text { PIS } & \text { positive ideal solution } \\ \text { NIS } & \text { negative ideal solution } \\ \text { FP } & \text { fuzzy programming } \\ \text { DM } & \text { decision maker }\end{array}$

\section{Problem description}

To transform homogeneous products from distinct sources to different destinations with certain condition in TP, we consider a multi-objective (here three objective functions) FSTP under budget constraints and on carbon emission in neutrosophic environment. The first objective function represents the profit over carbon emission charge and fixedcharge from each source to each destination. The second objective function is chosen as the deterioration rate of goods, and third one is taken as the transporting time of goods. To overcome the complexity of incomplete and indeterminate information, here we assume that all the parameters are NNs. The main aim is to obtain compromise optimal solution for transforming homogeneous products from $m$ sources to $n$ destinations using the $k$ conveyances at shipping cost $\tilde{c}_{i j k}^{n}$ per unit product in such a way that all the objective functions are optimized simultaneously. That is to shipping from supplier $i$ to customer $j$ by means of $k$ conveyance with fixed-charge $\tilde{f}_{i j k}^{n}$. Each supplier $(i=1,2, \ldots, m)$ has $\tilde{a}_{i}^{n}$ units of supply, each customer $(j=1,2, \ldots, n)$ has $\tilde{b}_{j}^{n}$ units of demand and each conveyance $(k=1,2, \ldots, l)$ has $\tilde{e}_{k}^{n}$ units of capacity.

\subsection{Notations of the proposed study}

The following notations are assumed to describe our proposed mathematical model as:

$x_{i j k}: \quad$ amount of product that to be transported from $i^{t h}$ source to $j^{\text {th }}$ destination with $k^{\text {th }}$ conveyance,

$\tilde{c}_{i j k}^{n}: \quad$ cost for unit quantity of the product from $i^{\text {th }}$ source to $j^{\text {th }}$ destination with $k^{\text {th }}$ conveyance,

$\tilde{f}_{i j k}^{n}: \quad$ fixed-charge for unit quantity of the product from $i^{\text {th }}$ source to $j^{\text {th }}$ destination with $k^{\text {th }}$ conveyance,

$\tilde{d}_{i j k}^{n}: \quad$ deterioration rate for unit quantity of the product from $i^{\text {th }}$ source to $j^{\text {th }}$ destination with $k^{\text {th }}$ conveyance,

$\tilde{t}_{i j k}^{n}: \quad$ time of transportation for unit quantity of the product from $i^{\text {th }}$ source to $j^{\text {th }}$ destination with $k^{\text {th }}$ conveyance,

$\eta\left(x_{i j k}\right)$ : binary variable takes the value " 1 " if $x_{i j k} \neq 0$ and " 0 " if $x_{i j k}=0$,

$\tilde{a}_{i}^{n}: \quad$ the supply at $i^{t h}$ source,

$\tilde{b}_{j}^{n}: \quad$ the demand at $j^{\text {th }}$ destination,

$\tilde{e}_{k}^{n}$ : the $k^{\text {th }}$ conveyance for TP,

$\tilde{B}_{j}^{n}$ : $\quad$ total budget at $j^{\text {th }}$ destination,

$\tilde{p}_{i}^{n}: \quad$ purchasing cost per unit quantity of product at $i^{t h}$ origin,

$\tilde{s}_{j}^{n}: \quad$ selling price per unit quantity of product at $j^{\text {th }}$ destination,

$\tilde{c}_{k}^{n}: \quad$ fixed carbon capacity,

$\tilde{e}_{i j k}^{n}: \quad$ charge of carbon emission per unit,

$\tilde{\alpha}^{n}: \quad$ carbon tax at per unit of its carbon emission $\left(\tilde{\alpha}^{n}>\right.$ 0 ),

$\tilde{Z}_{r}^{n}: \quad$ the objective function with NNs $(r=1,2,3)$,

$\bar{Z}_{r}^{\prime \prime}$ : $\quad$ the interval valued objective function $(r=1,2,3)$, $Z_{r}^{\prime L}: \quad$ the lower level objective function $(r=1,2,3)$ in crisp nature,

$Z_{r}^{\prime}: \quad$ the upper level objective function $(r=1,2,3)$ in crisp nature.

To obtain the maximum profit, it is obvious to minimize the purchasing cost, transportation cost, fixed-charge, carbon emission charge and maximize the selling price. Also in the same time, deterioration rate of goods and total delivery time of transportation must be minimized. Since there exist three objective functions which are contradict to each other and therefore the whole system is controlled by MOTP. Without loss of generality, we choose here first objective function becomes profit maximization, second objective function for minimizing deterioration and third objective func- 
tion is for time minimization. Again budget constraints and fixed carbon capacity are important features in our formulated model. These two constraints are also controlled the system to obtain maximum profit. The mathematical model for profit maximization multi-objective fixed-charge solid transportation problem (MFSTP) with carbon emission and budget constraints under neutrosophic environment is presented as:

\section{Model 1}

$\operatorname{maximize} \tilde{Z}_{1}^{n}=\sum_{i=1}^{m} \sum_{j=1}^{n} \sum_{k=1}^{l}\left[\left(\tilde{s}_{j}^{n}-\tilde{c}_{i j k}^{n}-\tilde{\alpha}^{n} \tilde{e}_{i j k}^{n}-\tilde{p}_{i}^{n}\right) x_{i j k}\right.$ $\left.-\tilde{f}_{i j k}^{n} \eta\left(x_{i j k}\right)\right]$

$\operatorname{minimize} \tilde{Z}_{2}^{n}=\sum_{i=1}^{m} \sum_{j=1}^{n} \sum_{k=1}^{l} \tilde{d}_{i j k}^{n} x_{i j k}$

$\operatorname{minimize} \tilde{Z}_{3}^{n}=\sum_{i=1}^{m} \sum_{j=1}^{n} \sum_{k=1}^{l} \tilde{t}_{i j k}^{n} \eta\left(x_{i j k}\right)$

subject to $\quad \sum_{j=1}^{n} \sum_{k=1}^{l} x_{i j k} \leq \tilde{a}_{i}^{n} \quad(i=1,2, \ldots, m)$,

$\sum_{i=1}^{m} \sum_{k=1}^{l} x_{i j k} \geq \tilde{b}_{j}^{n} \quad(j=1,2, \ldots, n)$,

$\sum_{i=1}^{m} \sum_{j=1}^{n} x_{i j k} \leq \tilde{e}_{k}^{n}(k=1,2, \ldots, l)$,

$\sum_{i=1}^{m} \sum_{k=1}^{l}\left[\tilde{p}_{i}^{n}+\tilde{c}_{i j k}^{n}+\tilde{\alpha}^{n} \tilde{e}_{i j k}^{n}\right] x_{i j k} \leq \tilde{B}_{j}^{n}$

$(j=1,2, \ldots, n)$,

$\sum_{i=1}^{m} \sum_{j=1}^{n} \tilde{e}_{i j k}^{n} x_{i j k} \leq \tilde{c}_{k}^{n}(k=1,2, \ldots, l)$,

$x_{i j k} \geq 0, \forall i, j, k$,

$\eta\left(x_{i j k}\right)=\left\{\begin{array}{l}1, \text { if } x_{i j k}>0 \\ 0, \text { otherwise. }\end{array}\right.$

The feasibility condition of this TP is as follows:

$\sum_{i=1}^{m} \tilde{a}_{i}^{n} \geq \sum_{j=1}^{n} \tilde{b}_{j}^{n} ; \sum_{k=1}^{l} \tilde{e}_{k}^{n} \geq \sum_{j=1}^{n} \tilde{b}_{j}^{n}$

Remark 1: All the parameters in the objective functions and constraints are NNs and based on this fact, Model 1 is treated as neutrosophic MFSTP. To derive the Pareto-optimal solution (compromise solution) of this model we consider that minimize $\tilde{Z}_{1}^{\prime}{ }_{1}=\operatorname{maximize}\left(-\tilde{Z}_{1}^{n}\right)$, minimize $\tilde{Z}_{2}^{\prime}{ }_{2}=$ minimize $\tilde{Z}_{2}^{n}$ and minimize $\tilde{Z}_{3}^{\prime}=\operatorname{minimize} \tilde{Z}_{3}^{n}$.

Theorem 3.1: Model 1 can be solved as multi-objective linear programming problem under NNs.

Proof: Model 1 satisfies all of the conditions stated in Definition 2.4. Therefore, Model 1 can be solved as general linear programming problem under neutrosophic environment. This evinces the proof of the theorem.
3.2 Equivalent deterministic form of Model 1

Without loss of generality, we choose the NN in Model 1 of the form $\tilde{a}^{n}=p+q I$, where $\mathrm{I}$ is the indeterminacy and then Model 1 transforms into Model 2 as:

\section{Model 2}

$$
\begin{aligned}
& \operatorname{minimize} \bar{Z}_{1}^{\prime n}=\sum_{i=1}^{m} \sum_{j=1}^{n} \sum_{k=1}^{l}\left[\left(p_{i}^{1}+I_{p} p_{i}^{2}\right)+\left(c_{i j k}^{1}+I_{c} c_{i j k}^{2}\right)\right. \\
& \left.+\left(\alpha^{1}+I_{\alpha} \alpha^{2}\right)\left(e_{i j k}^{1}+I_{e} e_{i j k}^{2}\right)-\left(s_{j}^{1}+I_{s} s_{j}^{2}\right)\right] x_{i j k} \\
& +\sum_{i=1}^{m} \sum_{j=1}^{n} \sum_{k=1}^{l}\left(f_{i j k}^{1}+I_{f} f_{i j k}^{2}\right) \eta\left(x_{i j k}\right) \\
& \operatorname{minimize} \bar{Z}_{2}^{\prime n}=\sum_{i=1}^{m} \sum_{j=1}^{n} \sum_{k=1}^{l}\left(d_{i j k}^{1}+I_{d} d_{i j k}^{2}\right) x_{i j k} \\
& \operatorname{minimize} \bar{Z}_{3}^{\prime n}=\sum_{i=1}^{m} \sum_{j=1}^{n} \sum_{k=1}^{l}\left(t_{i j k}^{1}+I_{t} t_{i j k}^{2}\right) \eta\left(x_{i j k}\right) \\
& \text { subject to } \sum_{j=1}^{n} \sum_{k=1}^{l} x_{i j k} \leq\left(a_{i}^{1}+I_{a} a_{i}^{2}\right) \quad(i=1,2, \ldots, m) \text {, } \\
& \sum_{i=1}^{m} \sum_{k=1}^{l} x_{i j k} \geq\left(b_{j}^{1}+I_{b} b_{j}^{2}\right)(j=1,2, \ldots, n), \\
& \sum_{i=1}^{m} \sum_{j=1}^{n} x_{i j k} \leq\left(e_{k}^{1}+I_{e} e_{k}^{2}\right) \quad(k=1,2, \ldots, l), \\
& \sum_{i=1}^{m} \sum_{k=1}^{l}\left[\left(p_{i}^{1}+I_{p} p_{i}^{2}\right)+\left(c_{i j k}^{1}+I_{c} c_{i j k}^{2}\right)+\left(\alpha^{1}+\right.\right. \\
& \left.\left.I_{\alpha} \alpha^{2}\right)\left(e_{i j k}^{1}+I_{e} e_{i j k}^{2}\right)\right] x_{i j k} \leq\left(B_{j}^{1}+I_{B} B_{j}^{2}\right) \\
& (j=1,2, \ldots, n) \text {, } \\
& \sum_{i=1}^{m} \sum_{j=1}^{n}\left(e_{i j k}^{1}+I_{e} e_{i j k}^{2}\right) x_{i j k} \leq\left(c_{k}^{1}+I_{c} c_{k}^{2}\right) \\
& (k=1,2, \ldots, l), \\
& x_{i j k} \geq 0, \forall i, j, k \text {, } \\
& \eta\left(x_{i j k}\right)=\left\{\begin{array}{l}
1, \text { if } x_{i j k}>0, \\
0, \text { otherwise. }
\end{array}\right.
\end{aligned}
$$

Now a NN can be expressed as $\tilde{a}^{n}=\left[p+q I^{L}, p+q I^{U}\right]=$ $\left[a^{L}, a^{U}\right]$ which becomes an interval number, and then there exist lower bound and upper bound of the interval. Therefore for existence of these bounds, Model 2 splits into two equivalent crisp problems, which are noted as Model $3 \mathrm{~A}$ and Model 3B. Model 3A chooses as lower level problem whereas Model 3B considers as upper level problem. Also using inequality 2.4.7. the interval valued constraints transform into deterministic form. Hence the deterministic form of these models are as follows: 


$$
\begin{aligned}
& \text { Model 3A } \\
& \operatorname{minimize} Z_{1}^{\prime L}=\sum_{i=1}^{m} \sum_{j=1}^{n} \sum_{k=1}^{l}\left[\left(p_{i}^{U}+c_{i j k}^{U}+\alpha^{U} e_{i j k}^{U}-s_{j}^{L}\right) x_{i j k}\right. \\
& \left.+f_{i j k}^{U} \eta\left(x_{i j k}\right)\right] \\
& \operatorname{minimize} Z_{2}^{L}=\sum_{i=1}^{m} \sum_{j=1}^{n} \sum_{k=1}^{l} d_{i j k}^{L} x_{i j k} \\
& \operatorname{minimize} Z_{3}^{\prime L}=\sum_{i=1}^{m} \sum_{j=1}^{n} \sum_{k=1}^{l} t_{i j k}^{L} \eta\left(x_{i j k}\right) \\
& \text { subject to } \quad \sum_{j=1}^{n} \sum_{k=1}^{l} x_{i j k} \leq a_{i}^{U} \quad(i=1,2, \ldots, m) \text {, } \\
& \sum_{i=1}^{m} \sum_{k=1}^{l} x_{i j k} \geq b_{j}^{L}(j=1,2, \ldots, n), \\
& \sum_{i=1}^{m} \sum_{j=1}^{n} x_{i j k} \leq e_{k}^{U} \quad(k=1,2, \ldots, l), \\
& \sum_{i=1}^{m} \sum_{k=1}^{l}\left[p_{i}^{L}+c_{i j k}^{L}+\alpha^{L} e_{i j k}^{L}\right] x_{i j k} \leq B_{j}^{L} \\
& (j=1,2, \ldots, n), \\
& \sum_{i=1}^{m} \sum_{k=1}^{l}\left[p_{i}^{U}+c_{i j k}^{U}+\alpha^{U} e_{i j k}^{U}\right] x_{i j k} \leq B_{j}^{U} \\
& (j=1,2, \ldots, n), \\
& \sum_{i=1}^{m} \sum_{j=1}^{n} e_{i j k}^{L} x_{i j k} \leq c_{k}^{L} \quad(k=1,2, \ldots, l), \\
& \sum_{i=1}^{m} \sum_{j=1}^{n} e_{i j k}^{U} x_{i j k} \leq c_{k}^{U} \quad(k=1,2, \ldots, l), \\
& x_{i j k} \geq 0, \forall i, j, k \text {, } \\
& \eta\left(x_{i j k}\right)=\left\{\begin{array}{l}
1, \text { if } x_{i j k}>0, \\
0, \text { otherwise. }
\end{array}\right.
\end{aligned}
$$

\section{Model 3B}

$\operatorname{minimize} Z_{1}^{\prime U}=\sum_{i=1}^{m} \sum_{j=1}^{n} \sum_{k=1}^{l}\left[\left(p_{i}^{L}+c_{i j k}^{L}+\alpha^{L} e_{i j k}^{L}-s_{j}^{U}\right) x_{i j k}\right.$

$$
\left.+f_{i j k}^{L} \eta\left(x_{i j k}\right)\right]
$$

$\operatorname{minimize} Z_{2}^{\prime U}=\sum_{i=1}^{m} \sum_{j=1}^{n} \sum_{k=1}^{l} d_{i j k}^{U} x_{i j k}$

$\operatorname{minimize} Z_{3}^{\prime}=\sum_{i=1}^{m} \sum_{j=1}^{n} \sum_{k=1}^{l} t_{i j k}^{U} \eta\left(x_{i j k}\right)$

subject to constraints (4) $-(12)$.

Henceforth the definition of Pareto-optimal solution (compromise solution) is defined as follows.

Definition 3.1: Pareto-optimal solution (compromise solution) of Model 3A/Model 3B is a feasible solution $x^{*}=$ $\left(x_{i j k}^{*}: i=1,2, \ldots, m ; j=1,2, \ldots, n ; k=1,2, \ldots, l\right)$ such that there exists no other feasible solution $x=\left(x_{i j k}: i=1,2, \ldots, m\right.$; $j=1,2, \ldots, n ; k=1,2, \ldots, l)$ with $Z_{r}^{\prime}(x) \leq Z_{r}^{\prime}\left(x^{*}\right), r=1,2,3$ and $Z_{r}^{\prime}(x)<Z_{r}^{\prime}\left(x^{*}\right)$ for at least one $r$.

Theorem 3.2: The combination of the Pareto-optimal solutions of Model 3A and Model 3B represent the Paretooptimal solution of Model 2 and consequently of Model 1 in the form of interval neurtrosophic number.

Proof: Using Definition 2.5, Theorem 3.2 can be proven easily.

\section{Solution methodology}

In multi-objective optimization problem, there does not always exist a solution which is the best for all the objective functions. That is the solution will be the best for one objective function and that may be worst for another objective function. The objective functions are conflicting to each other and hence the solutions cannot simply compare to each other. For this cause, we discuss three methods for solving neutrosophic MFSTP as follows:

- Neutrosophic linear programming problem (NLPP),

- Fuzzy programming (FP),

- Global criterion method.

\subsection{Neutrosophic linear programming problem (NLPP)}

Model 3A and Model 3B provide the lower bound and upper bound of the objective functions and the solutions are not overall compromise solution of Model 2. So we utilize NLPP to derive the compromise solution of multi-objective decision making problem. To solve the proposed model in NLPP, we describe the following steps as:

- Step 4.1.1: Transform the neutrosophic optimization problem into crisp problem which splits into two sub-problems.

- Step 4.1.2: Solve each problem individually with subject to all constraints.

- Step 4.1.3: Determine the upper bound as positive ideal solution (PIS) and lower bound as negative ideal solution (NIS) for each objective function in the pay-off matrix, displaying in Table 1, where PIS and NIS are defined as PIS $=Z_{r}{ }^{*}=\min \left\{Z_{r}\left(X_{1}{ }^{*}\right), Z_{r}\left(X_{2}{ }^{*}\right), Z_{r}\left(X_{3}{ }^{*}\right)\right\}(r=$ $1,2,3)$ and NIS $=Z_{r}^{\prime}=$ $\max \left\{Z_{r}\left(X_{1}^{*}\right), Z_{r}\left(X_{2}^{*}\right), Z_{r}\left(X_{3}^{*}\right)\right\}(r=1,2,3)$ respectively.

- Step 4.1.4: Design the truth-membership function and indeterminacy-membership function with highest degree and falsity-membership function with least degree.

Table 1 Pay-off matrix.

\begin{tabular}{|l|l|l|l|}
\hline & $Z_{1}$ & $Z_{2}$ & $Z_{3}$ \\
\hline$X_{1}{ }^{*}$ & $Z_{1}\left(X_{1}{ }^{*}\right)$ & $Z_{2}\left(X_{1}{ }^{*}\right)$ & $Z_{3}\left(X_{1}{ }^{*}\right)$ \\
$X_{2}{ }^{*}$ & $Z_{1}\left(X_{2}{ }^{*}\right)$ & $Z_{2}\left(X_{2}{ }^{*}\right)$ & $Z_{3}\left(X_{2}{ }^{*}\right)$ \\
$X_{3}{ }^{*}$ & $Z_{1}\left(X_{3}{ }^{*}\right)$ & $Z_{2}\left(X_{3}{ }^{*}\right)$ & $Z_{3}\left(X_{3}{ }^{*}\right)$ \\
\hline
\end{tabular}


- Step 4.1.5: Setting the tolerance and constructing the membership functions according to the bounds as:

$$
\begin{aligned}
& T_{l}\left(Z_{l}^{\prime}(x)\right)= \begin{cases}1, & \text { if } Z_{l}^{\prime}(x) \leq L_{l}^{T}, \\
1-\frac{Z_{l}^{\prime}(x)-L_{l}^{T}}{U_{l}^{T}-L_{l}^{T}}, & \text { if } L_{l}^{T} \leq Z_{l}^{\prime}(x) \leq U_{l}^{T}, \\
0, & \text { if } Z_{l}^{\prime}(x) \geq U_{l}^{T},\end{cases} \\
& I_{l}\left(Z_{l}^{\prime}(x)\right)= \begin{cases}0, & \text { if } Z_{l}^{\prime}(x) \leq L_{l}^{I}, \\
1-\frac{Z_{l}^{\prime}(x)-L_{l}^{I}}{U_{l}^{I}-L_{l}^{I}}, & \text { if } L_{l}^{I} \leq Z_{l}^{\prime}(x) \leq U_{l}^{I}, \\
0, & \text { if } Z_{l}^{\prime}(x) \geq U_{l}^{I},\end{cases} \\
& F_{l}\left(Z_{l}^{\prime}(x)\right)= \begin{cases}0, & \text { if } Z_{l}^{\prime}(x) \leq L_{l}^{F}, \\
1-\frac{Z_{l}^{\prime}(x)-L_{l}^{F}}{U_{l}^{F}-L_{l}^{F}}, & \text { if } L_{l}^{F} \leq Z_{l}^{\prime}(x) \leq U_{l}^{F}, \\
1, & \text { if } Z_{l}^{\prime}(x) \geq U_{l}^{F},\end{cases}
\end{aligned}
$$

Here $U_{l}^{T}=U_{l}=$ PIS for $Z_{l}^{\prime}$, and $L_{l}^{T}=L_{l}=$ NIS for $Z_{l}^{\prime}$; $U_{l}^{F}=U_{l}^{T}, L_{l}^{F}=L_{l}^{T}+t_{l}\left(U_{l}^{T}-L_{l}^{T}\right) ; L_{l}^{I}=L_{l}^{T}, U_{l}^{I}=L_{l}^{T}+$ $s_{l}\left(U_{l}^{T}-L_{l}^{T}\right) ; t_{l}, s_{l}$ are tolerances.

- Step 4.1.6: Choose the values of $\alpha, \beta, \gamma$ in $[0,1]$ for each neutrosophic number as the truth, indeterminacy and falsity degrees respectively.

- Step 4.1.7: Constitute NLPP that represents in Model 4A.

\section{Model 4A}

$\operatorname{maximize} T_{l}\left(Z_{l}^{\prime}(x)\right)(l=1,2,3)$

maximize $I_{l}\left(Z_{l}^{\prime}(x)\right)(l=1,2,3)$

minimize $F_{l}\left(Z_{l}^{\prime}(x)\right)(l=1,2,3)$

subject to constraints $(4)-(12)$.

Model 4A can be reduced to Model 4B as:

\section{Model 4B}

maximize $\alpha$

$\operatorname{maximize} \beta$

minimize $\gamma$

subject to $T_{l}\left(Z_{l}^{\prime}(x)\right) \geq \alpha, I_{l}\left(Z_{l}^{\prime}(x)\right) \geq \beta, F_{l}\left(Z_{l}^{\prime}(x)\right) \leq \gamma$ $\alpha+\beta+\gamma \leq 3, \alpha+\beta+\gamma \geq 0, \alpha \geq \gamma, \alpha \geq \beta$, $\alpha, \beta, \gamma \in[0,1],(l=1,2,3)$, constraints $(4)-(12)$.

Now the simplified model of NLPP (Model 4B) that derives the compromise solution of MOTP (i.e., Model 4C) as follows:

\section{Model 4C}

$$
\begin{aligned}
\operatorname{maximize} & \alpha+\beta-\gamma \\
\text { subject to } & Z_{l}^{\prime}(x)+\left(U_{l}^{T}-L_{l}^{T}\right) \alpha \leq U_{l}^{T}, \\
& Z_{l}^{\prime}(x)+\left(U_{l}^{I}-L_{l}^{I}\right) \beta \leq U_{l}^{I}, \\
& Z_{l}^{\prime}(x)-\left(U_{l}^{F}-L_{l}^{F}\right) \gamma \leq U_{l}^{F}, \\
& \alpha+\beta+\gamma \leq 3, \alpha+\beta+\gamma \geq 0, \alpha \geq \gamma, \alpha \geq \beta, \\
& \alpha, \beta, \gamma \in[0,1],(l=1,2,3), \\
& \text { constraints }(4)-(12) .
\end{aligned}
$$

- Step 4.1.8: Solve Model 4C by LINGO 13 iterative scheme.

Theorem 4.1: If $x^{*}=\left(x_{i j k}: i=1,2, \ldots, m ; j=1,2, \ldots, n ; k\right.$ $=1,2, \ldots, p)$ is an optimal solution of Model $4 \mathrm{C}$ then it is also Pareto-optimal (non-dominated) solution of Model 2.

Proof: Let $x^{*}$ is not a Pareto optimal (non-dominated) solution of Model 2. Therefore, from Def. 3.1, we consider that there exists at least one $x$ such that $Z_{l}^{\prime}(x) \leq Z_{l}^{\prime}\left(x^{*}\right)$ for $l=$ $1,2,3$ and $Z_{l}^{\prime}(x)<Z_{l}^{\prime}\left(x^{*}\right)$ for at least one $l$. Therefore truth and indeterminacy membership functions $\mu_{l}^{I}\left(Z_{l}^{\prime}(x)\right)$ and $\sigma_{l}^{I}\left(Z_{l}^{\prime}(x)\right)$ are strictly decreasing with respect to the corresponding objective function $Z_{l}$ in $[0,1]$ respectively. Again the falsity membership function $v_{l}^{I}\left(Z_{l}^{\prime}(x)\right)$ strictly increases with respect to the objective function $Z_{l}^{\prime}$ in $[0,1]$. Hence $\mu_{l}^{I}\left(Z_{l}^{\prime}(x)\right) \geq \mu_{l}^{I}\left(Z_{l}^{\prime}\left(x^{*}\right)\right) \forall l$ and $\mu_{l}^{I}\left(Z_{l}^{\prime}(x)\right)>\mu_{l}^{I}\left(Z_{l}^{\prime}\left(x^{*}\right)\right)$ for at least one $l$. Similarly $\sigma_{l}^{I}\left(Z_{l}^{\prime}(x)\right) \geq \sigma_{l}^{I}\left(Z_{l}^{\prime}\left(x^{*}\right)\right) \forall l$ and $\sigma_{l}^{I}\left(Z_{l}^{\prime}(x)\right)>\sigma_{l}^{I}\left(Z_{l}^{\prime}\left(x^{*}\right)\right)$ for at least one $l$. Also $v_{l}^{I}\left(Z_{l}^{\prime}(x)\right) \leq$ $v_{l}^{I}\left(Z_{l}^{\prime}\left(x^{*}\right)\right) \forall l$ and $v_{l}^{I}\left(Z_{l}^{\prime}(x)\right)<v_{l}^{I}\left(Z_{l}^{\prime}\left(x^{*}\right)\right)$ for at least one $l$. Now, $(\alpha+\beta-\gamma)=\min \left\{\mu_{l}^{I}\left(Z_{l}^{\prime}(x)\right), \sigma_{l}^{I}\left(Z_{l}^{\prime}(x)\right), v_{l}^{I}\left(Z_{l}^{\prime}(x)\right)\right\}$ $\geq \min \left\{\mu_{l}^{I}\left(Z_{l}^{\prime}\left(x^{*}\right)\right), \sigma_{l}^{I}\left(Z_{l}^{\prime}\left(x^{*}\right)\right), v_{l}^{I}\left(Z_{l}^{\prime}\left(x^{*}\right)\right)\right\}=\left(\alpha^{*}+\beta^{*}-\right.$ $\left.\gamma^{*}\right)$ which is a contradiction that $x^{*}$ is an optimal solution of Model 4C. Here $\alpha^{*}, \beta^{*}$ and $\gamma^{*}$ are the values of $\alpha, \beta$ and $\gamma$ at $x^{*}$ respectively. This completes the proof of the theorem.

\subsection{Fuzzy programming (FP)}

Since Models 3A and 3B provide the lower bound and upper bound of the objective functions and the solutions are not overall compromise solution of Model 2. Therefore to find overall compromise solution, we take the advantage of FP which is used to solve neutrosophic MFSTP. FP was introduced by Zimmermann (1978) for solving multi-objective linear programming problem and it is very easy for solving this type of problem. FP of Model 2 can be formulated as: Find $x=\left(x_{1}, x_{2}, \ldots, x_{n}\right)^{T}$ such that minimize $Z_{l}^{\prime}(l=1,2,3)$ and subject to $g_{j}(X) \leq 0,(j=1,2, \ldots, m)$ and $x_{i} \geq 0,(i=$ $1,2, \ldots, n)$, with tolerance $a_{l}(l=1,2,3)$. The membership function $\mu_{l}^{I}\left(Z_{l}^{\prime}(x)\right)(l=1,2,3)$ which is defined in Def. 2.2. Our goal is to maximize the degree of acceptance of objective functions. Therefore to solve the proposed Model 2 in FP, we depict the following steps as:

- Step 4.2.1: Transform the neutrosophic optimization problem into crisp problem which becomes into two subproblems.

- Step 4.2.2: Solve each problem independently with subject to all constraints.

- Step 4.2.3: Select the tolerance of each objective function.

- Step 4.2.4: Determine PIS and NIS defined in Step 4.1.3. and formulate the membership function corresponding to each objective function. Now maximize the degree of acceptance of objective function and then the equivalent 
crisp model of FP is designed as:

Model 5A

maximize $\alpha$

subject to $T_{l}\left(Z_{l}^{\prime}(x)\right) \geq \alpha(l=1,2,3)$,

$\alpha \in[0,1]$, constraints $(4)-(12)$.

Model 5A is transformed into simplified form in Model 5B as:

\section{Model 5B}

maximize $\alpha$

subject to $Z_{l}^{\prime}(x)+\left(U_{l}^{T}-L_{l}^{T}\right) \alpha \leq U_{l}^{T}(l=1,2,3)$,

$\alpha \in[0,1]$,

constraints $(4)-(12)$.

- Step 4.2.6: Solve Model 5B by LINGO 13 iterative scheme with respect to parameter $\alpha$.

Theorem 4.2: If $x^{*}=\left(x_{i j k}: i=1,2, \ldots, m ; j=1,2, \ldots, n\right.$; $k=1,2, \ldots, p)$ is an optimal solution of Model $5 \mathrm{~B}$ then it is also Pareto-optimal (non-dominated) solution of Model 2.

Proof: Let $x^{*}$ is not a Pareto optimal (non-dominated) solution of Model 2. Therefore, from Def. 3.1, we consider that there exists at least one $x$ such that $Z_{l}^{\prime}(x) \leq Z_{l}^{\prime}\left(x^{*}\right)$ for $l=$ $1,2,3$ and $Z_{l}^{\prime}(x)<Z_{l}^{\prime}\left(x^{*}\right)$ for at least one $l$. Therefore membership function $\mu_{l}^{I}\left(Z_{l}^{\prime}(x)\right)$ is strictly decreasing with respect to the corresponding objective function $Z_{l}^{\prime}$ in $[0,1]$. Hence $\mu_{l}^{I}\left(Z_{l}^{\prime}(x)\right) \geq \mu_{l}^{I}\left(Z_{l}^{\prime}\left(x^{*}\right)\right) \forall l$ and $\mu_{l}^{I}\left(Z_{l}^{\prime}(x)\right)>\mu_{l}^{I}\left(Z_{l}^{\prime}\left(x^{*}\right)\right)$ for at least one $l$. Now, $\alpha=\min \left\{\mu_{l}^{I}\left(Z_{l}^{\prime}(x)\right)\right\} \geq \min \left\{\mu_{l}^{I}\left(Z_{l}^{\prime}\left(x^{*}\right)\right)\right\}$ $=\alpha^{*}$ which is a contradiction that $x^{*}$ is an optimal solution of Model 5B. Here $\alpha^{*}$ is the value of $\alpha$ at $x^{*}$. This completes the proof of the theorem.

\subsection{Global criterion method}

Here we introduce another approach, namely, global criterion method that provides the compromise solution by minimizing distance among some reference points of the feasible objective region. Since Models 3A and 3B do not provide overall compromise solution of Model 2. Therefore to find overall compromise solution, global criterion method of Model 2 can be depicted by the following steps:

- Step 4.3.1: Transform the neutrosophic optimization problem into crisp problem which becomes into two subproblems.

- Step 4.3.2: Solve each problem independently with subject to all constraints.

- Step 4.3.3: Determine the $\max \left(U_{l}^{T}\right)$ and $\min \left(L_{l}^{T}\right)$ value of each objective function from Table 1 and formulate the crisp model is as:

\section{Model 6}

minimize $F(x)=\left[\sum_{l=1}^{3}\left(\frac{Z_{l}^{\prime}(x)-L_{l}^{T}}{U_{l}^{T}-L_{l}^{T}}\right)^{2}\right]^{\frac{1}{2}}$

subject to constraints (4) - (12).

- Step 4.3.4: Solve Model 6 by LINGO 13 iterative scheme.

Definition 3.2: The compromise solution of global criterion method is defined as the minimum distance between the ideal solution and the desired solution. If $Z_{l}{ }^{*}$ is the ideal solution of the objective function $Z_{l}^{\prime}$ then the compromise solution of Model 2 is defined as $Z_{l}^{*}=\min \left\|Z_{l}^{\prime *}-Z_{l}^{\prime}\right\|_{\infty} \forall l$.

\subsection{Differences among solution methodologies}

First of all, global criterion method is a non-fuzzy technique i.e., this method does not take into account indeterminacy in the optimization problems. Therefore, we need not have to construct any membership or non-membership functions, only we have to build a single objective optimization problem utilizing different norms such as $L_{1}, L_{2}, L_{\infty}$, etc.. On the other hand, FP is a fuzzy optimization technique which considers vagueness in optimization problems. Therefore, in FP we have to build membership function for the objective functions. Again, NLPP is an extended version of FP, which handles the indeterminacy occupied into the optimization problem in neutrosophic manner i.e., in NLPP we have to construct three membership functions namely truth, falsity and indeterminacy.

\section{Limitations of the study}

From literature review, we observe that many researchers formulated STP/MFSTP in different environments such as fuzzy and IF optimization for the parameters of cost, time and deterioration. But their formulated models have some limitations which are listed here.

- Li and Lai (2000) solved MOTP using fuzzy programming for controlling the uncertainty. Majumder et al. (2019) solved uncertain multi-item MFSTP with budget constraints. This problem was analyzed with the uncertainty for determining truth and falsity but they did not think about indeterminacy that enrolled the system into neutrosophic.

- Rizk-Allah et al. (2018) used neutrosophic compromise programming for solving MOTP, without considering neutrosophic number for the parameters of MOTP. In neutrosophic system, there exists indeterminacy membership function which is also an important factor for uncertainty. 
- Ding et al. (2013) included about carbon emission in transportation system and its potential for reduction in China, but it was not connected with other factors in multi-level system such as profit, budget, carbon capacity, fixed-charge etc. which are indirectly related with carbon emission.

- Tarulescu et al. (2017) discussed about smart transportation $\mathrm{CO}_{2}$ emission reduction strategies but did not consider about carbon capacity, budget and profit which are indirectly related in transportation system with fuzzy, intuitionistic fuzzy or neutrosophic environment.

- Das et al. (2016) solved breakable multi-stage multiitem STP under budget with Gaussian type-2 fuzzy parameters but did not include with neutrosophic system.

- Khalil et al. (2018) discussed more on time neutrosophic soft set and its applications but we see that the paper was defined only fundamental definitions, examples and operations on time neutrosophic set without discussing about the real-life application or any programming problem.

- Song and Leng (2012) analyzed about single-period problem under carbon emission policies but did not incorporate multi-level or multi-period problem and not defined for fuzzy or intuitionistic fuzzy or neutrosophic environment.

\section{Advances of the proposed study}

- Neutrosophic sets are characterised by three independent membership degrees, nam-ely, truth-membership degree (T), indeterminacy-membership degree (I) and falsitymembership degree $(F)$ which are more capable to handle imprecise parameters.

- IFSs can only handle the incomplete information not indeterminate. But neutrosophic set can tackle both incomplete information and indeterminate information. Therefore the neutrosophic set is more applicable for complete uncertainty than the IFS.

- Decision makers (DMs) in neutrosophic set want to increase the degree of truth-membership function and the degree of indeterminacy but decrease the degree of falsitymembership function, which are more realistic in reallife problem.

- Relating with transportation system, carbon emission increases the rate of air pollution and thereafter increases the carbon emission charge. Hence profit of the system decreases and therefore to obtain maximum profit, carbon emission reduction is an important factor which are chosen in this paper.
- Also to obtain maximum profit, budget constraint and carbon capacity are included in this problem and these extra restrictions that help to find minimum deterioration and minimum time of the proposed problem.

\section{Real-life Experiment}

In this section, we include a real-life example to illustrate the applicability of the proposed approach with maximum profit, minimum deterioration and minimum time in transportation system. Considering two source points of West Bengal that export two types of sea fishes to other states (e.g., Bihar and Jharkhand) in transportation system. For heavy duty of vehicle in transportation system, carbon emission charge includes with other costs such as selling price, purchasing cost, transportation cost, fixed-charge. Again for conservation strategy of data, deterioration cost is included. Also for transporting data with long distance, time factor must be added. According to various complicated factors, supply, demand and conveyance are taken as NNs. Transportation cost per unit item from source to destination with fixed-charge, deterioration cost, time, carbon emission charge are all NNs which are supplied in Tables 2, 3, 4 and 5 respectively. Transportation cost, deterioration cost and carbon emission charge are considered in hundred dollar (\$) per unit and time in hour per unit. Also the selling price, purchasing cost, carbon tax, source, demand, conveyance, budget and carbon capacity which are NNs are chosen here. The aim is to obtain maximum profit by minimizing purchasing cost, carbon emission charge, transportation charge and fixed-charge, and maximizing selling price. Time and deterioration are also minimized such that the budget and carbon capacity must be bounded. Therefore the problem becomes MFSTP due to describe of such conditions with the objective functions which are contradicted to each other. The formulation of mathematical form of this problem are Models 7A and 7B which are obtained from Models 3A and 3B respectively. Solution of the problem is now illustrated thereafter. In addition to that we assume the following in the proposed study.

Selling price $=\left(\tilde{s}_{j}^{n}\right):\left\{\tilde{s}_{1}^{n}=50+5 \mathrm{I} ; \tilde{s}_{2}^{n}=60+5 \mathrm{I}\right\}$; Purchasing cost $=$ $\left(\tilde{p}_{i}^{n}\right):\left\{\tilde{p}_{1}^{n}=5+\mathrm{I} ; \tilde{p}_{2}^{n}=6+2 \mathrm{I}\right\} ;$ Carbon tax $=\left(\tilde{\alpha}^{n}\right):\{\alpha=2+I\}$; Source $=\left(\tilde{a}_{i}^{n}\right):\left\{\tilde{a}_{1}^{n}=230+5 \mathrm{I} ; \tilde{a}_{2}^{n}=240+10 \mathrm{I}\right\} ;$ Demand $=\left(\tilde{b}_{j}^{n}\right)$ : $\left\{\tilde{b}_{1}^{n}=100+5 \mathrm{I} ; \tilde{b}_{2}^{n}=120+10 \mathrm{I}\right\} ;$ Conveyance $=\left(\tilde{e}_{k}^{n}\right):\left\{\tilde{e}_{1}^{n}=270+5 \mathrm{I}\right.$; $\left.\tilde{e}_{2}^{n}=290+10 \mathrm{I}\right\}$; Budget $=\left(\tilde{B}_{j}^{n}\right):\left\{\tilde{B}_{1}^{n}=3900+100 \mathrm{I} ; \tilde{B}_{2}^{n}=3500\right.$ $+100 \mathrm{I}\}$; Carbon capacity $=\left(\tilde{c}_{k}^{n}\right):\left\{\tilde{c}_{1}^{n}=360+40 \mathrm{I} ; \tilde{c}_{2}^{n}=420+40 \mathrm{I}\right\}$. Here we consider $I=[0,1]$ is the indeterminacy. 
Table 2 Neutrosophic transportation cost $\tilde{c}_{i j k}^{n}$ and the fixed-charge $\tilde{f}_{i j k}^{n}$.

\begin{tabular}{|l|l|l|l|}
\hline & $D_{1}$ & $D_{2}$ & $a_{i}$ \\
\hline & $k=1$ & $k=2$ & \\
\hline$c_{11 k}$ & $(6+4 I)$ & $(8+3 I)$ & $(230+5 I)$ \\
$c_{12 k}$ & $(15+2 I)$ & $(4+I)$ & \\
$f_{11 k}$ & $(10+8 I)$ & $(9+7 I)$ & \\
$f_{12 k}$ & $(25+2 I)$ & $(7+2 I)$ & \\
\hline$c_{21 k}$ & $(4+2 I)$ & $(10+4 I)$ & $(240+10 I)$ \\
$c_{22 k}$ & $(3+I)$ & $(5+2 I)$ & \\
$f_{21 k}$ & $(6+2 I)$ & $(13+10 I)$ & \\
$f_{22 k}$ & $(7+2 I)$ & $(15+2 I)$ & \\
\hline$b_{j}$ & $(100+5 I)$ & $(120+10 I)$ & \\
\hline
\end{tabular}

Table 3 Neutrosophic deterioration cost $\tilde{d}_{i j k}^{n}$.

\begin{tabular}{|l|l|l|l|}
\hline & $D_{1}$ & $D_{2}$ & $a_{i}$ \\
\hline & $k=1$ & $k=2$ & \\
\hline$d_{11 k}$ & $(4+2 I)$ & $(5+2 I)$ & $(230+5 \mathrm{I})$ \\
$d_{12 k}$ & $(5+I)$ & $(3+2 I)$ & \\
\hline$d_{21 k}$ & $(3+I)$ & $(4+2 I)$ & $(240+10 \mathrm{I})$ \\
$d_{22 k}$ & $(2+I)$ & $(2+I)$ & \\
\hline$b_{j}$ & $(100+5 \mathrm{I})$ & $(120+10 \mathrm{I})$ & \\
\hline
\end{tabular}

Table 4 Neutrosophic time $\tilde{t}_{i j k}^{n}$.

\begin{tabular}{|l|l|l|l|}
\hline & $D_{1}$ & $D_{2}$ & $a_{i}$ \\
\hline & $k=1$ & $k=2$ & \\
\hline$t_{11 k}$ & $(12+3 I)$ & $(15+5 I)$ & $(230+5 \mathrm{I})$ \\
$t_{12 k}$ & $(8+2 I)$ & $(11+4 I)$ & \\
\hline$t_{21 k}$ & $(6+4 I)$ & $(9+5 I)$ & $(240+10 \mathrm{I})$ \\
$t_{22 k}$ & $(10+5 I)$ & $(14+6 I)$ & \\
\hline$b_{j}$ & $(100+5 \mathrm{I})$ & $(120+10 \mathrm{I})$ & \\
\hline
\end{tabular}

Table 5 Carbon emission charge $\tilde{e}_{i j k}^{n}$.

\begin{tabular}{|l|l|l|l|}
\hline & $D_{1}$ & $D_{2}$ & $a_{i}$ \\
\hline & $k=1$ & $k=2$ & \\
\hline$e_{11 k}$ & $(2+4 I)$ & $(3+3 I)$ & $(230+5 \mathrm{I})$ \\
$e_{12 k}$ & $(3+2 I)$ & $(2+I)$ & \\
\hline$e_{21 k}$ & $(2+3 I)$ & $(2+I)$ & $(240+10 \mathrm{I})$ \\
$e_{22 k}$ & $(2+2 I)$ & $(3+2 I)$ & \\
\hline$b_{j}$ & $(100+5 \mathrm{I})$ & $(120+10 \mathrm{I})$ & \\
\hline
\end{tabular}

\section{Model 7A}

minimize $Z_{1}^{\prime L}=2 x_{111}+x_{112}+5 x_{121}-31 x_{122}-13 x_{211}$

$$
+4 x_{212}-27 x_{221}-13 x_{222}
$$

minimize $Z_{2}^{\prime L}=4 x_{111}+5 x_{112}+5 x_{121}+3 x_{122}+3 x_{211}$

$$
+4 x_{212}+2 x_{221}+2 x_{222}
$$

minimize $Z_{3}^{\prime L}=12 x_{111}+15 x_{112}+8 x_{121}+11 x_{122}+6 x_{211}$

$$
+9 x_{212}+10 x_{221}+14 x_{222}
$$

subject to $\quad x_{111}+x_{112}+x_{121}+x_{122} \leq 235$,

$x_{211}+x_{212}+x_{221}+x_{222} \leq 250$,

$x_{111}+x_{112}+x_{211}+x_{212} \geq 100$,

$x_{121}+x_{122}+x_{221}+x_{222} \geq 120$,

$x_{111}+x_{121}+x_{211}+x_{221} \leq 275$,

$x_{112}+x_{122}+x_{212}+x_{222} \leq 300$,
Table 6 Pay off matrix for $Z_{l}^{\prime L}(l=1,2,3)$.

\begin{tabular}{|l|l|l|l|}
\hline & $Z_{1}^{\prime L}$ & $Z_{2}^{\prime L}$ & $Z_{3}^{L}$ \\
\hline$X_{1}{ }^{L}$ & $-5093.23^{*}$ & $719.99^{\prime}$ & 2126.63 \\
$X_{2}{ }^{L}$ & $-2550.77^{\prime}$ & $621.54^{*}$ & $2216.92^{\prime}$ \\
$X_{3}{ }^{L}$ & -4680 & 680 & $1980^{*}$ \\
\hline
\end{tabular}

Table 7 Pay off matrix for $Z_{l}^{\prime}(l=1,2,3)$.

\begin{tabular}{|l|l|l|l|}
\hline & $Z_{1}^{\prime U}$ & $Z_{2}^{\prime}{ }^{U}$ & $Z_{3}^{\prime}$ \\
\hline$X_{1}{ }^{U}$ & $-9239.85^{*}$ & 1106.65 & 3079.95 \\
$X_{2}{ }^{U}$ & -7323.08 & $923.08^{*}$ & $3341.54^{\prime}$ \\
$X_{3}{ }^{U}$ & $-5706.52^{\prime}$ & $1240.01^{\prime}$ & $2813.33^{*}$ \\
\hline
\end{tabular}

$\leq 4000$,

$13 x_{111}+19 x_{112}+14 x_{211}+20 x_{212}$

$\leq 3900$,

$38 x_{121}+20 x_{122}+24 x_{221}+30 x_{222}$

$\leq 3600$,

$26 x_{121}+13 x_{122}+13 x_{221}+17 x_{222}$

$\leq 3500$,

$2 x_{111}+3 x_{121}+2 x_{211}+2 x_{221} \leq 360$,

$6 x_{111}+5 x_{121}+5 x_{211}+4 x_{221} \leq 400$,

$3 x_{112}+2 x_{122}+2 x_{212}+3 x_{222} \leq 420$,

$6 x_{112}+3 x_{122}+3 x_{212}+5 x_{222} \leq 460$,

$x_{i j k} \geq 0, \forall i, j, k$.

\section{Model 7B}

$$
\begin{aligned}
& \operatorname{minimize} Z_{1}^{\prime U}=-30 x_{111}-27 x_{112}-14 x_{121}-45 x_{122} \\
&-35 x_{211}-22 x_{212}-45 x_{221}-33 x_{222} \\
& \operatorname{minimize} Z_{2}^{\prime U}= 6 x_{111}+7 x_{112}+6 x_{121}+5 x_{122}+4 x_{211} \\
&+6 x_{212}+3 x_{221}+3 x_{222} \\
& \text { minimize } Z_{3}^{\prime U}= 15 x_{111}+20 x_{112}+10 x_{121}+15 x_{122}+10 x_{211} \\
&+14 x_{212}+15 x_{221}+20 x_{222} \\
& \text { subject to } \quad \text { constraints }(13)-(27) .
\end{aligned}
$$

Solving Model 7A and Model 7B using LINGO 13 iterative scheme, we derive the solutions and calculate the value of the objective functions that are reflected in Tables 6 and 7 . Since the solutions are contradict to each other and to find the overall compromise solution we solve Model 4C with the help of proposed NLPP. The PIS and NIS are obtained from Tables 6 and 7 and they are denoted by $*$ and ' respectively. Finally we design Model 8 with help of NLPP to find the compromise solution of Model 2. 


\section{Model 8}

maximize $\alpha+\beta-\gamma$

subject to $Z_{l}^{\prime L}(x)+\left(U_{l}^{L}-L_{l}^{L}\right) \alpha \leq U_{l}^{L}$,

$Z_{l}^{\prime L}(x)+\left(U_{l}^{L}-L_{l}^{L}\right)(\beta-1) s_{1 l} \leq L_{l}^{L}$,

$Z_{l}^{\prime L}(x)-\left(U_{l}^{L}-L_{l}^{L}\right) \gamma\left(1-t_{1 l}\right) \leq U_{l}^{L}$,

$Z_{l}^{\prime U}(x)+\left(U_{l}^{U}-L_{l}^{U}\right) \alpha \leq U_{l}^{U}$,

$Z^{\prime}{ }_{l}^{U}(x)+\left(U_{l}^{U}-L_{l}^{U}\right)(\beta-1) s_{2 l} \leq L_{l}^{U}$,

$Z_{l}^{\prime U}(x)-\left(U_{l}^{U}-L_{l}^{U}\right) \gamma\left(1-t_{2 l}\right) \leq U_{l}^{U}$,

$\alpha+\beta+\gamma \leq 3, \alpha \geq \gamma, \alpha \geq \beta$,

$\alpha, \beta, \gamma \in[0,1],(l=1,2,3)$

constraints $(13)-(27)$.

Where $U_{l}^{\mathrm{L}}=\mathrm{PIS}$ of $Z_{l}^{\mathrm{L}}, L_{l}^{\mathrm{L}}=\mathrm{NIS}$ of $Z_{l}^{\mathrm{L}} ; U_{l}^{\mathrm{U}}=\mathrm{PIS}$ of $Z_{l}^{\mathrm{U}}$, $L_{l} \mathrm{U}=\mathrm{NIS}$ of $Z_{l} \mathrm{U}$. The solutions for Model 8 are obtained as: $\alpha=1, \beta=1, \gamma=0.002368 ; x_{122}=59.08542, x_{211}=$ $54.82179, x_{212}=45.17821, x_{221}=31.47276, x_{222}=29.442$, $x_{111}=x_{112}=x_{121}=0 ; Z_{1}^{L}=6323.33, Z_{2}^{L}=644.26, Z_{3}^{L}=$ $50 ; Z_{1}^{U}=9294.01, Z_{2}^{U}=963.53, Z_{3}^{U}=74$.

Therefore the compromise solutions of proposed Model 1 in NLPP is $Z_{1}=[6323.33,9294.01], Z_{2}=[644.26,963.53]$, $Z_{3}=[50,74]$. Also the solutions of FP and global criterion methods are respectively given as: $Z_{1}=[5900.82,9482.29]$, $Z_{2}=[719.97,1048.82], Z_{3}=[58,84]$; and $Z_{1}=[5906.9$, $11941.13], Z_{2}=[720.49,1056.57], Z_{3}=[52,74]$.

\section{- Results and discussion}

Solving Model 2 with help of NLPP, FP and global criterion method, and the solutions of Model 1 are depicted in Section 7. Therefore the values of three objective functions obtained by three methods are displayed in Table 8 as:

Table 8 Solution of NLPP, FP and Global criteria method.

\begin{tabular}{|l|l|l|l|}
\hline Methods & $Z_{1}$ & $Z_{2}$ & $Z_{3}$ \\
\hline NLPP & $(6323.33,9294.01)$ & $(644.26,963.53)$ & $(50,74)$ \\
FP & $(5900.82,9482.29)$ & $(719.97,1048.82)$ & $(58,84)$ \\
Global Criteria & $(5906.9,11941.13)$ & $(720.49,1056.57)$ & $(52,74)$ \\
\hline
\end{tabular}

Comparing the results that calculated from the NLPP, FP and global criterion method, we conclude that optimal values of the objective functions $\left(Z_{1}, Z_{2}, Z_{3}\right)$ that received from NLPP always provides better result than FP and global criterion method. Also, from the analysis, it is very much essential to include the extra restriction on carbon capacity in our formulated problem to safe our environment and to obtain maximum profit in transportation system by minimizing the total transportation cost.

\section{Sensitivity analysis}

To explain and interpret the effect of change to the coefficients of the objective functions, sensitivity analysis is a compulsive and concerning procedure in optimization problem. Now, it is difficult to analyze the range of all parametric values and its slide change such that the optimal value remains same. There exist some research papers on sensitive analysis about transportation problem with linear programming, such as Hasen et al. (1989), Ebrahimnejad (2011) etc. But whenever there exist large change of variables and constraints, then a complexity arises such that the values of basic variables are changed. Therefore to control over these complexity, we introduce sensitivity analysis of MFSTP with NLPP such that all the basic variables remain fixed. Now to find the ranges of these parameters in neutrosophic MFSTP, we define the steps as follows:

- Step 8.1: Taking all the basic variables as fixed for the optimal solution of MFSTP.

- Step 8.2: Change the values of each parameter at a time with fixing other parameters and solve the MFSTP by LINGO 13 iterative scheme.

- Step 8.3: Continue Step 8.2, until change the basic variable or no feasible solution arises in optimal solution.

- Step 8.4: Find the range of each parameter in Step 8.3. Sensitivity analysis for supply, demand and conveyance parameters changes as:

Let $a_{i}$ change to $a_{i}^{*}$ as $a_{i}^{*}=a_{i}+\theta_{i},(i=1,2), b_{j}$ change to $b_{j}^{*}$ as $b_{j}^{*}=b_{j}+\eta_{j},(j=1,2)$ and $e_{k}$ change to $e_{k}^{*}$ as $e_{k}^{*}=e_{k}+\tau_{k},(k=1,2)$. Using the described procedure, we derive the values of $a_{i}^{*}, b_{j}^{*}$, and $e_{k}^{*}$ which are shown in Table 9.

Table 9 Ranges of supply, demand and conveyance.

\begin{tabular}{|l|l|}
\hline Actual values of $a_{i}, b_{j}$ and $e_{k}$ & Changes values of $a_{i}, b_{j}$ and $e_{k}$ \\
\hline$a_{1}=230$ & $10.5 \leq a_{1}^{*}<\infty$ \\
$a_{2}=240$ & $120.1 \leq a_{2}^{*}<\infty$ \\
$b_{1}=100$ & $94.4 \leq b_{1}^{*} \leq 115.1$ \\
$b_{2}=120$ & $113.6 \leq b_{2}^{*} \leq 136.6$ \\
$e_{1}=270$ & $80.3 \leq e_{1}^{*}<\infty$ \\
$e_{2}=290$ & $120.1 \leq e_{2}^{*}<\infty$ \\
\hline
\end{tabular}

\section{Conclusion and future research scopes}

Transportation system always depends on various factors. The most of the common factors are time, profit, budget, deterioration, carbon emission, purchasing cost, selling price, fixed-charge etc. Since all the data are collected from real world system, therefore there exist some complexity, restriction and uncertainty. The aim of our transportation problem is to overcome such complexity by taking neutrosophic data and find the compromise solution. Here we take all the data as NNs and use NLPP that maximizes the truth-membership value, the indeterminacy-membership value and minimizes 
falsity-membership value. Again carbon emission is included and we see that this improves the profit, reduce the air pollution which is more necessary. Three methods have been executed to obtain the compromise solutions by solving the multi-objective transportation problem. Out of which NLPP has provided the best solution and the method is also very simple, suitable and applicable for multi-objective decision making problem. Fuzzy set and IFS only find the uncertainty with membership and non-membership value, but neutrosophic system adds an extra important aspect as indeterminacy membership value which is independent on membership and non-membership values. Therefore neutrosophic system is more reliable than fuzzy system or IFS for clearly defining incomplete and indeterminate information on reallife problems. The applicability of the proposed approach has been clarified through a real-life example. Moreover, the stable ranges of some parameters have been revealed by the sensitivity analysis. Finally, some decisions regarding the budget and carbon emission during transportation have been discussed which can be very helpful to the organizations/companies for resolving the economical and environmental issues.

In future scope of research, neutrosophic system can be extended as bipolar-neutrosophic system and can be applied for linear or non-linear problem. Also carbon emission system will be taken as cap and trade policy with neutrosophic number. Neutrosophic programming with neutrosophic number can be applied in fractional problem or multi-item transportation. Different uncertainties such as type-2 neutrosophic, type-2 uncertain variable, uncertain-random, type- 2 intuitionistic fuzzy, etc. can be developed in our model. Furthermore, several heuristics, meta-heuristics and hybrid methods can be developed to solve the large instances of our proposed problem.

Acknowledgement: The authors are very much grateful to the Editor-in-Chief and anonymous respective reviewer for the insightful comments that helped us so much to rigorously improve the quality of the manuscript. The author Sankar Kumar Roy is very thankful to Jose Luis Verdegay for giving the opportunity to complete the work during the visit to the Department of Computer Science and Artificial Intelligence, University of Granada, Granada, Spain in the month July, 2019.

\section{Compliance with ethical standards}

Conflict of Interest: The authors would like to announce that there is no conflict of interest.

Ethical approval: This article does not contain any studies with human participants or animals performed by any of the authors.

Funding: Research supported in part by project PID2020112754GB-I00 (Spanish Govern and FEDER funds from the European Union )

\section{References}

Atanassov KT (1986) Intuitionistic fuzzy sets. Fuzzy Sets Syst. 20(1):87 - 96 .

Das A, Bera UK, Maiti M (2016) A breakable multiitem multi stage solid transportation problem under budget with gaussian type-2 fuzzy parameters. Appl. Intell. 45(3):923-951.

Das SK, Roy SK (2019) Effect of variable carbon emission in a multi-objective transportation-p-facility location problem under neutrosophic environment. Comput. Ind. Eng. 132:311-324.

Das SK, Roy SK, Weber GW (2020) Application of type-2 fuzzy logic to a multiobjective green solid transportationlocation problem with dwell time under carbon tax, cap, and offset policy: Fuzzy versus nonfuzzy techniques. IEEE Trans. Fuzzy Syst. 28(11):2711-2725.

Ding J, Jin F, Li Y, Wang J (2013) Analysis of transportation carbon emissions and its potential for reduction in china. Chinese J. Population Res. Env. 11(1):17-25.

Ebrahimnejad A (2014) A simplified new approach for solving fuzzy transportation problems with generalized trapezoidal fuzzy numbers. Appl. Soft Comput. 19:171-176.

Ghosh S, Roy SK (2021) Fuzzy-rough multi-objective product blending fixed-charge transportation problem with truck load constraints through transfer station. RairoOper. Res. 55: S2923-S29527.

Ghosh S, Roy SK, Ebrahimnejad A, Verdegay JL (2021) Multi-objective fully intuitionistic fuzzy fixed-charge solid transportation problem. Complex Intell. Syst. 7(2):1009-1023.

Haley K (1962) New methods in mathematical programming - the solid transportation problem. Oper. Res. 10(4):448-463.

Hirsch WM, Dantzig GB (1968) The fixed charge problem. Nav. Res. Logist. Q. 15(3):413-424.

Hitchcock FL (1941) The distribution of a product from several sources to numerous localities. J Math. Phys. 20(14):224-230.

Ishibuchi H, Tanaka H (1990) Multiobjective programming in optimization of the interval objective function. Eur. J. Oper. Res. 48(2):219-225.

Khalil AM, Alkhazaleh S, Li SG, You F, Ma SQ (2018) More on "time-neutrosophic soft set and its applications". J. Intell. Fuzzy Syst. 35(5):5721-5728.

Li L, Lai KK (2000) A fuzzy approach to the multiobjective transportation problem. Comput. Oper. Res. 27(1):43-57.

Maity G, Roy SK, Verdegay JL (2016) Multi-objective transportation problem with cost reliability under uncertain environment. Int. J. Comput. Intell. Syst. 9(5):839849.

Maity G, Roy SK, Verdegay JL (2019) Analyzing multimodal transportation problem and its application to artifi- 
cial intelligence. Neural Comput. Appl. 32:2243-2256.

Majumder S, Kundu P, Kar S, Pal T (2019) Uncertain multi-objective multi-item fixed charge solid transportation problem with budget constraint. Soft Comput. 23(10):3279-3301.

Midya S, Roy SK (2014) Solving single-sink, fixedcharge, multi-objective, multi-index stochastic transportation problem. American J. Math. Manag. Sci. 33(4):300-314.

Midya S, Roy SK (2017) Analysis of interval programming in different environments and its application to fixedcharge transportation problem. Discrete Math. Algorithms Appl., 9(03):1750040.

Midya S, Roy SK, Vincent FY (2021) Intuitionistic fuzzy multi-stage multi-objective fixed-charge solid transportation problem in a green supply chain. Int. J. Mach. Learn. Cybern. 12(3):699-717.

Rizk-Allah RM, Hassanien AE, Elhoseny M (2018) A multi-objective transportation model under neutrosophic environment. Comput. Elect. Eng. 69:705-719.

Roy SK, Midya S (2019) Multi-objective fixed-charge solid transportation problem with product blending under intuitionistic fuzzy environment. Appl. Intell. 49(10):35243538.

Roy SK, Midya S, Weber GW (2019) Multi-objective multiitem fixed-charge solid transportation problem under twofold uncertainty. Neural Comput. Appl. 31(12):85938613.

Roy SK, Midya S, Yu VF (2018) Multi-objective fixedcharge transportation problem with random rough variables. Int. J. Uncert. Fuzziness Knowl.-Based. Syst. 26(06):971-996.

Sengupta D, Das A, Bera UK (2018) A gamma type2 defuzzification method for solving a solid transportation problem considering carbon emission. Appl. Intell. 48(11):3995-4022.

Sifaoui1 T, Aider M (2020) Uncertain interval programming model for multi-objective multi-item fixed charge solid transportation problem with budget constraint and safety measure. Soft Comput. 24: 10123-10147.

Smarandache F (1999) A unifying field in logics. neutrosophy: Neutrosophic probability, set and logic.

Smarandache F (2013) Introduction to neutrosophic measure, neutrosophic integral, and neutrosophic probability. Infinite Study.

Song J, Leng M (2012) Analysis of the single-period problem under carbon emissions policies. In Handbook of newsvendor problems, pages 297-313. Springer.

Tarulescu S, Tarulescu R, Soica A, Leahu CI (2017) Smart transportation $\mathrm{CO}_{2}$ emission reduction strategies. In IOP Conf. Series: Mater. Sci. Eng., volume 252, page 012051. IOP Publishing.
Wang H, Smarandache F, Zhang Y, Sunderraman R (2010) Single valued neutrosophic sets. Infinite study.

Ye J (2018) Neutrosophic number linear programming method and its application under neutrosophic number environments. Soft comput. 22(14):4639-4646.

Ye J, Cui W, Lu Z (2018) Neutrosophic number nonlinear programming problems and their general solution methods under neutrosophic number environments. Axioms 7(1):13.

Zadeh LA (1965) Fuzzy sets. Inf. Control 8(3):338-353.

Zimmermann HJ (1978) Fuzzy programming and linear programming with several objective functions. Fuzzy Sets Syst. 1(1):45-55. 